\title{
CFTR Modulation by the Tobacco Smoke Toxin Acrolein
}

\author{
Nathan S. Alexander, M.D. ${ }^{1}$, Angela Blount, M.D., Shaoyan Zhang, Ph.D. ${ }^{1,2}$, Daniel Skinner, \\ B.S. ${ }^{1,2}$, Stephen B. Hicks, B.S. ${ }^{1}$, Michael Chestnut, B.S. ${ }^{1}$, Frederick A. Kebbel, B.S. ${ }^{1}$, Eric J. \\ Sorscher, M.D. ${ }^{2}$, and Bradford A. Woodworth, M.D. ${ }^{1,2}$ \\ ${ }^{1}$ Department of Surgery/Division of Otolaryngology, University of Alabama at Birmingham, AL \\ ${ }^{2}$ Gregory Fleming James Cystic Fibrosis Research Center, University of Alabama at Birmingham, \\ $\mathrm{AL}$
}

\begin{abstract}
Objectives-Evidence indicates that decreased mucociliary clearance (MCC) is a major contributing feature to chronic rhinosinusitis. Tobacco-smoke exposure is thought to inhibit transepithelial $\mathrm{Cl}^{-}$secretion - a major determinant of airway surface liquid hydration and MCC. The objective of the current study was to evaluate the effects of acrolein exposure (a prominent tobacco smoke toxin) on vectorial $\mathrm{Cl}^{-}$transport through the major apical anion channel CFTR in sinonasal epithelium.
\end{abstract}

Study Design-In vitro investigation.

Methods-Primary murine nasal septal (MNSE, wild type and transgenic $\mathrm{CFTR}^{--}$) cultures were exposed to acrolein in Ussing chambers and effects on $\mathrm{Cl}^{-}$secretion investigated using pharmacologic manipulation. Cellular cAMP signaling and cytotoxicity were also investigated.

Results-Acrolein stimulated $\mathrm{Cl}^{-}$secretion $\left(\Delta \mathrm{I}_{\mathrm{SC}}-\right.$ change in short-circuit current in $\left.\mu \mathrm{A} / \mathrm{cm}^{2}\right)$ at concentrations similar to smoker's airways $(100 \mu \mathrm{M}, 15.8+/-2.2$ vs. $2.4+/-0.8$ (control); $\mathrm{p}<0.0001)$, suppressed forskolin-stimulated $\mathrm{Cl}^{-}$transport at $300 \mu \mathrm{M}(13.3+/-1.2 \mathrm{vs.} 19.9+/-$ $1.0 ; \mathrm{p}<0.01\}$, and completely abolished all transport at $500 \mu \mathrm{M}(-1.1+/-1.6)$. Stimulated $\mathrm{Cl}^{-}$ secretion was solely reliant upon the presence of CFTR (confirmed in transgenic $\mathrm{CFTR}^{-/-}$ MNSE), but independent of cAMP signaling. Inhibition at higher concentrations was not secondary to cellular cytotoxicity.

Conclusions-The present study demonstrates that acrolein has complex, but pronounced interaction with the major apical $\mathrm{Cl}^{-}$transport mechanism that utilizes CFTR. Further investigations are required to determine acrolein's impact as a tobacco smoke constituent on mucociliary transport.

\section{Keywords}

Tobacco; cigarette smoke; acrolein; sinusitis; CFTR; chloride; cAMP; LDH; electrophysiology; Ussing chamber

Corresponding Author Bradford A. Woodworth, M.D., BDB 563, $15303^{\text {rd }}$ Ave S, Birmingham, AL 35294, 205-934-9765 (Phone), 205-934-3993 (Fax), bwoodwo@hotmail.com.

Conflict of Interest/Financial Disclosures: Bradford A. Woodworth, MD is a consultant for ArthroCare ENT and Gyrus ENT. Eric Sorscher, MD, and Bradford Woodworth, MD are inventors on a patent pending regarding the use of chloride secretagogues for therapy of sinus disease (35 U.S.C. n111(b) and 37 C.F.R n.53 (c) in the U.S. Patent and Trademark Office.

This manuscript was presented at the AAO-HNS annual meeting, San Francisco, September 12, 2011. 


\section{INTRODUCTION}

Sinonasal respiratory epithelium maintains a highly regulated mucociliary apparatus that clears inhaled pathogens from the nose and paranasal sinuses. The airway surface liquid (ASL) is a crucial part of the mucociliary apparatus, and its composition is determined by the transepithelial vectorial transport of ions, such as chloride $\left(\mathrm{Cl}^{-}\right)$and sodium $\left(\mathrm{Na}^{+}\right) .{ }^{1}$ One of the primary regulators of $\mathrm{Cl}^{-}$transport in sinonasal respiratory epithelium is the cystic fibrosis transmembrane conductance regulator (CFTR). Dysfunctional $\mathrm{Cl}^{-}$transport through acquired (e.g., toxic exposures, hypoxia) or genetic (i.e., cystic fibrosis) disease can lead to dehydration of the ASL, stasis of inspissated mucus, bacterial infection, and widespread chronic sinus disease. ${ }^{2-4}$

Cigarette smokers exhibit a higher rate of recurrent sinusitis and chronic rhinosinusitis (CRS) when compared to nonsmokers. ${ }^{5}$ Chronic smoking is known to contribute to immunosuppressive changes (decreased lipopolysaccharide-induced inflammatory cytokines and suppression of human monocyte-derived macrophage function) impairing host defenses and increasing susceptibility to infection. ${ }^{6}$ Cigarette smoke is a highly toxic substance that is composed of a complex mixture of over 5,000 chemicals, including several that have been identified as having high toxicity to respiratory cilia - specifically acrolein, formaldehyde, carbon monoxide, nicotine, cotinine, acetaldehyde, phenol, and potassium cyanide. ${ }^{3,6-9}$

Acrolein is one of the most abundant volatile, electrophilic compounds present in cigarette smoke and is known to elicit several different biochemical responses including transcription factor activation, oxidative injury, inflammation, alteration of immunologic function, ciliotoxicity, and cell death. ${ }^{10}$ Sarkar et al. ${ }^{11}$ demonstrated that one cigarette contains approximately 238-468ug acrolein, and that acrolein induces an inflammatory response through the rapid reduction of glutathione, protein sulfhydruls, and thiol-containing enzymes.

The effects of cigarette smoke on sinonasal epithelium are thought in part to be secondary to impairment of ciliary function and dehydration of the ASL. ${ }^{12-15}$ In a prior study by our group, cigarette smoke condensate (CSC), which contains the particulate and waterinsoluble components of tobacco combustion, was shown to substantially decrease transepithelial transport of $\mathrm{Cl}^{-}$, and to strongly impair ciliary beat frequency. ${ }^{3}$ Others have reported suppression with the water soluble extract of cigarette smoke suggesting that a number of agents in tobacco could be responsible for reduction in mucociliary transport. ${ }^{16}$ While the inflammatory and immunologic effects of acrolein have been investigated previously, no reports to date have examined its impact on transepithelial $\mathrm{Cl}^{-}$secretion. The present study was designed to investigate the effects of acrolein on vectorial $\mathrm{Cl}^{-}$transport through CFTR, the major apical anion channel in sinonasal epithelium. Experiments were designed to test the hypothesis that acrolein reduces transepithelial CFTR-mediated $\mathrm{Cl}^{-}$ secretion.

\section{MATERIALS AND METHODS}

Institutional review board and institutional animal care and use committee approval were obtained prior to the initiation of the study.

\section{Tissue Culture}

The culture technique for propagation of murine nasal septal epithelia (MNSE) at an airliquid interface has been described previously. ${ }^{2,17-22}$ Briefly, tissue was harvested and grown on Costar 6.5-mm-diameter permeable filter supports (Corning Life Sciences, Lowell, MA) submerged in culture medium. The media was removed from the surface of the 
monolayers on day 4 and the cells fed via the basal chamber. MNSE cultures were genetically identical and derived from the C57/BL6 strain and from transgenic CFTR ${ }^{-/-}$ knockout mice. Differentiation and ciliogenesis occurred in all cultures within 10 days to 14 days. Ussing chamber analysis was performed only when cell monolayers were fully differentiated with widespread ciliogenesis and transepithelial resistances $\left(R_{T}\right)>500 \Omega \mathrm{cm}^{2}$. There were no significant differences between the $\mathrm{R}_{\mathrm{T}}$ and $\mathrm{I}_{\mathrm{SC}}$ of control and acroleinexposed filters when the two groups were compared before the addition of acrolein, or pharmacologic agents.

\section{Electrophysiology}

Solutions and chemicals-The bath solution contained (mM): $120 \mathrm{NaCl}, 25 \mathrm{NaHCO}_{3}$, $3.3 \mathrm{KH}_{2} \mathrm{PO}_{4}, 0.8 \mathrm{~K}_{2} \mathrm{HPO}_{4}, 1.2 \mathrm{MgCl}_{2}, 1.2 \mathrm{CaCl}_{2}$, and 10 glucose, with pH 7.3-7.4 when gassed in a mixture of $95 \% \mathrm{O}_{2}-5 \% \mathrm{CO}_{2}$ at $37^{\circ} \mathrm{C}$. Chemicals were obtained from Sigma (St. Louis, MO). Each solution was made as $1,000 \mathrm{x}$ stock and used at $1 \mathrm{x}$ in the Ussing chamber. All experiments were performed with low $\mathrm{Cl}^{-}(6 \mathrm{mM})$ in the mucosal bath. Pharmacologic preparations were as follows: amiloride $(100 \mu \mathrm{M})$; forskolin $(20 \mu \mathrm{M})$; acrolein $(1 \mu \mathrm{M}$ to 500 $\mu \mathrm{M})$; $\mathrm{CFTR}_{\mathrm{INH}^{-1}} 172(10 \mu \mathrm{M})$; amphotericin $(50 \mu \mathrm{M})$. Amiloride blocks $\mathrm{Na}^{+}$channels, ensuring that change in short-circuit current $\left(\Delta \mathrm{I}_{\mathrm{SC}}\right)$ from subsequent manipulations are secondary to effects on $\mathrm{Cl}^{-}$channel activity. Forskolin activates the cystic fibrosis transmembrane conductance regulator (CFTR) via a cyclic adenosine monophosphate (cAMP)-mediated mechanism. CFTR $\mathrm{INH}^{-172}$ is a very specific inhibitor of CFTR and was used to develop comparisons of the relative activity of CFTR in different systems. Acrolein is a water soluble component of cigarette smoke and, as such, was dissolved in water for all experiments.

Short circuit (Isc) measurements-Transwell inserts (Costar, Corning Life Sciences) were mounted in Ussing chambers to investigate ion transport using pharmacologic blockade and stimulation of plasma membrane ion channels. Monolayers were continuously short-circuited after fluid resistance compensation using automatic VCC 600 voltage clamps (Physiologic Instruments, San Diego, CA). Transwell filters were mounted in bath solution warmed to $37^{\circ} \mathrm{C}$, and the solution gas-lifted with a $95 \% \mathrm{O}_{2} / 5 \% \mathrm{CO}_{2}$ mixture. The $\mathrm{I}_{\mathrm{SC}}$ was measured at 1 sample per second. A positive deflection in $\mathrm{I}_{\mathrm{SC}}$ is defined as the net movement of anion in the serosal to mucosal direction. All experiments were performed using $\geq 5$ filters per condition.

Cellular cAMP Assay-Activation of CFTR anion transport requires protein kinase A (PKA)-dependent phosphorylation of the regulatory domain. An ELISA-based detection kit (Cayman Chemicals, Ann Arbor, MI) was used to measure stimulation of cellular cAMP by acrolein in MNSE cultures, as previously described. ${ }^{23}$

Assessment of Cell Viability-Membrane integrity of cells was quantified by detection of lactate dehydrogenase in the medium using an in vitro toxicology assay kit (Sigma, Catalog Number TOX7). Media was collected after cells were treated apically for 15 minutes with either acrolein $(100 \mu \mathrm{M}, 300 \mu \mathrm{M}, 500 \mu \mathrm{M})$ or PBS control. Absorbance was measured at a wavelength of $490 \mathrm{~nm}$ and subtracted from the background absorbance (690 $\mathrm{nm})$ at analysis.

Insert summary-A total of 103 MNSE filters were used in the completion of these studies with $n \geq 4$ per measured condition. MNSE filters were derived from genetically identical C57 mice or CFTR ${ }^{-/-}$transgenic mice as stated. Analyses were performed when cell monolayers were fully differentiated with widespread ciliogenesis and transepithelial resistances $\left(R_{t}\right)>500 \Omega . \mathrm{cm}^{2}$. There was no significant difference between the $R_{t}$ and $I_{S C}$ of 
control and acrolein-exposed filters when groups were compared before the addition of pharmacologic agents.

Statistical Analyses-Statistical analyses were performed using two-tailed paired $t$ test, unpaired $t$ test, and analysis of variance, where applicable.

\section{RESULTS}

\section{Activation and inhibition of CFTR-mediated $\mathrm{Cl}^{-}$transport is concentration dependent}

In dose-dependent studies, acrolein stimulated $\mathrm{Cl}^{-}$secretion $\left(\Delta \mathrm{I}_{\mathrm{SC}}-\right.$ change in short-circuit current in $\mu \mathrm{A} / \mathrm{cm}^{2}$ ) at a concentration expected in the setting of cigarette smoke inhalation in vivo $(100 \mu \mathrm{M}, 15.9+/-2.2$ vs. $2.4+/-0.8$ (control); $\mathrm{p}<0.0001)$, but suppressed total apical $\mathrm{Cl}^{-}$secretion (acrolein + forskolin) at $300 \mu \mathrm{M}(13.3+/-1.2$ vs. $19.9+/-1.0 ; \mathrm{p}<0.01\}$, and completely abolished all transport at $500 \mu \mathrm{M}(-1.1+/-1.6)$. (Figure $1 \& 2)$ There were no significant differences in measurements of total stimulation $(21.7+/-1.6$ vs. $19.9+/-0.1$; control) or CFTR inhibition with $\mathrm{CFTR}_{\mathrm{INH}^{-172}}$ (-14.8 +/- 0.5 vs. $-16.3+/-0.9$; control) with exposure to the $100 \mu \mathrm{M}$ concentration (Figure 3). Stimulated $\mathrm{Cl}^{-}$secretion was solely reliant upon the presence of CFTR (confirmed in transgenic CFTR -/- MNSE, Figure 4).

\section{Acrolein does not modulate CFTR through cAMP-mediated signal transduction}

Modulation of CFTR related effects were independent of cAMP-signaling (expressed as $\mathrm{pmol} / \mathrm{ml})$ as there were no significant cAMP elevations $(100 \mu \mathrm{M}, 1.4+/-0.2 ; 300 \mu \mathrm{M}, 1.3$ $+/-0.6 ; 500 \mu \mathrm{M}, 1.4+/-0.2)$ compared to controls [PBS (- control), $0.9+/-0.2$ and forskolin (+ control), 217.6 +/- $2.1(20 \mathrm{mM})$ and $18.4+/-1.5(100 \mathrm{nM})]$. (Figure 5) Importantly, pre-incubation with 300 or $500 \mu \mathrm{M}$ acrolein did not inhibit cAMP stimulation by forskolin. In fact, administration of $100 \mathrm{nM}$ forskolin after $300 \mu \mathrm{M}$ acrolein resulted in a significant increase in cAMP over $100 \mathrm{nM}$ forskolin alone (36.4 +/- 3.6 vs. 18.4 +/- 1.5, respectively).

\section{CFTR-mediated effects are not reflective of acute cytotoxicity}

Cell membrane integrity was not compromised by acute exposures to acrolein at any concentrations tested as judged by LDH release assay. The result is concordant with the lack of effect due to acrolein on tissue monolayer resistance in Ussing chamber (data not shown) which is often used as a proxy for cytotoxic drug effects. The negative impact of acrolein on CFTR dependent $\mathrm{I}_{\mathrm{SC}}$ measured at higher concentrations is therefore not secondary to cytotoxicity.

\section{DISCUSSION}

The present studies explored effects of the cigarette smoke toxin acrolein on transepithelial $\mathrm{Cl}^{-}$secretion and underlying cellular cAMP in a well established murine primary nasal cell culture model. Our prior investigations have shown that cigarette smoke condensate (CSC) inhibits major components of mucociliary transport, including transepithelial CFTRmediated $\mathrm{Cl}^{-}$secretion, and ciliary beat in MNSE and human sinonasal epithelial (HSNE) cell cultures. ${ }^{3}$ Additionally, we demonstrated previously that CSC inhibits calcium activated chloride channel $(\mathrm{CaCC})$ - mediated transepithelial $\mathrm{Cl}^{-}$secretion, and have confirmed this finding in transgenic CFTR ${ }^{-/-}$airway epithelial monolayers. ${ }^{14}$ These earlier findings lend support to the hypothesis that cigarette smoke suppresses apical $\mathrm{Cl}^{-}$secretion in respiratory epithelium and leads to ASL dehydration and dysfunctional MCC similar to cystic fibrosis airways. However, the specific components and the mechanism by which cigarette smoke suppresses $\mathrm{Cl}^{-}$secretion have not previously been determined. 
At a dose $(100 \mu \mathrm{M})$ that approximates the concentration found in the in vivo airways of tobacco smokers, ${ }^{10,24}$ acrolein significantly stimulated apical $\mathrm{Cl}^{-}$secretion. While cigarette smoke contains hundreds of toxins, acrolein by itself appears to promote hypersecretion at acute physiologic concentrations. Thus, inhibition of CFTR-mediated Cl- transport by water-soluble extract of cigarette smoke as previously reported is not mediated by this constituent. However, paradoxical inhibition of $\mathrm{Cl}^{-}$transport was demonstrated at higher test doses. Expression of CFTR is necessary to promote $\mathrm{Cl}^{-}$secretion through the pathway influenced by acrolein, since Ussing chamber tracings from transgenic $\mathrm{CFTR}^{-/-}$MNSE led to complete loss of this secretory mechanism. While CFTR-mediated pathways are stimulated, in part, by cAMP-dependent signaling, acrolein did not promote activation of adenylyl cyclase at any concentration. In addition, the suppression of forskolin-mediated activation of CFTR seen at higher concentrations of acrolein was not attributable to inhibitory effects on cellular cAMP. Importantly, no cytotoxic effects were demonstrated at the concentrations and exposures tested in the current investigations, indicating inhibitory effects at high concentrations are not due to cytotoxicity. While physiologic doses of acrolein led to an increase in CFTR-mediated $\mathrm{Cl}^{-}$transport, our data provide the first evidence of direct ion transport regulation by a cigarette smoke constituent. Paradoxical stimulation and inhibition have been demonstrated with other CFTR channel potentiating agents, such as flavonoids, in the absence of cAMP-mediated signaling and suggests that acrolein could interact with nucleotide-binding domains of CFTR in a similar fashion. ${ }^{2,14,21}$ Further studies, including patch clamp recordings, will be necessary to determine whether acrolein binds directly to CFTR or utilizes an alternative signaling mechanism to exert its effects on transepithelial $\mathrm{Cl}^{-}$transport.

Exposure to acrolein results in complex consequences for respiratory epithelial cells, including direct damage due to formation of adducts with proteins (disrupting cell structure or function) or nucleic acids (eliciting mutagenic or carcinogenic effects), and when effects such as depletion of antioxidants (e.g. glutathione) rendering a cell prone to damage from free radicals. ${ }^{25}$ Furthermore, expression of CFTR gene, protein, and function in response to cigarette smoke extract in vitro ${ }^{16,26}$ and our previous studies in MNSE and HSNE ${ }^{3,14}$ indicate deleterious consequences for mucociliary transport. While, the present findings establish that acute incubations with acrolein at physiologic concentrations suggest that hypersecretion would be more evident in the airways of individuals with acute exposure to this toxin, future investigations will examine prolonged exposures at physiologic concentrations and other tobacco smoke constituents for CFTR-related effects on mucociliary clearance.

\section{CONCLUSION}

The present study demonstrated that acrolein has complex, but significant interactions with CFTR, the major apical $\mathrm{Cl}^{-}$conductance pathway in sinonasal epithelium. The findings describe a means by which the effects of CFTR modulation by cigarette smoke can be better understood in the future.

\section{Acknowledgments}

Research Support: This research was funded by the National Institutes of Health/National Heart, Lung, and Blood Institute (1K08HL107142-01) and Flight Attendant's Medical Research Institute Young Clinical Scientist Award (072218) to B.A.W.; and NIH/NIDDK (5P30DK072482-03) to E.J.S. 


\section{REFERENCES}

1. Trout L, King M, Feng W, Inglis SK, Ballard ST. Inhibition of airway liquid secretion and its effect on the physical properties of airway mucus. Am J Physiol. 1998; 274:L258-L263. [PubMed: 9486211]

2. Alexander NS, Hatch N, Zhang Set al. Resveratrol has salutary effects on mucociliary transport and inflammation in sinonasal epithelium. Laryngoscope. 2011; 121:1313-1319. [PubMed: 21480283]

3. Cohen NA, Zhang S, Sharp DB, et al. Cigarette smoke condensate inhibits transepithelial chloride transport and ciliary beat frequency. Laryngoscope. 2009

4. Blount A, Zhang S, Chestnut M, et al. Transepithelial ion transport is suppressed in hypoxic sinonasal epithelium. Laryngoscope. 2011; 121:1929-1934. [PubMed: 22024847]

5. Lieu JE, Feinstein AR. Confirmations and surprises in the association of tobacco use with sinusitis. Arch Otolaryngol Head Neck Surg. 2000; 126:940-946. [PubMed: 10922224]

6. Lee WK, Ramanathan M Jr, Spannhake EW, Lane AP. The cigarette smoke component acrolein inhibits expression of the innate immune components IL- 8 and human beta-defensin 2 by sinonasal epithelial cells. Am J Rhinol. 2007; 21:658-663. [PubMed: 18201443]

7. Agius AM, Smallman LA, Pahor AL. Age, smoking and nasal ciliary beat frequency. Clin Otolaryngol. 1998; 23:227-230. [PubMed: 9669071]

8. Dalhamn T, Rylander R. Ciliotoxicity of cigar and cigarette smoke. Arch Environ Health. 1970; 20:252-253. [PubMed: 5411395]

9. Dalhamn T, Rylander R. Tar content and ciliotoxicity of cigarette smoke. Acta Pharmacol Toxicol (Copenh). 1967; 25:369-372. [PubMed: 5630494]

10. Zemski Berry KA, Murphy RC. Characterization of acrolein-glycerophosphoethanolamine lipid adducts using electrospray mass spectrometry. Chem Res Toxicol. 2007; 20:1342-1351. [PubMed: 17636891]

11. Sarkar P, Hayes BE. Induction of COX-2 by acrolein in rat lung epithelial cells. Mol Cell Biochem. 2007; 301:191-199. [PubMed: 17318410]

12. Moodie FM, Marwick JA, Anderson CS, et al. Oxidative stress and cigarette smoke alter chromatin remodeling but differentially regulate NF-kappaB activation and proinflammatory cytokine release in alveolar epithelial cells. Faseb J. 2004; 18:1897-1899. [PubMed: 15456740]

13. Verra F, Escudier E, Lebargy F, Bernaudin JF, De Cremoux H, Bignon J. Ciliary abnormalities in bronchial epithelium of smokers, ex-smokers, and nonsmokers. Am J Respir Crit Care Med. 1995; 151:630-634. [PubMed: 7881648]

14. Virgin FW, Azbell C, Schuster Det al. Exposure to cigarette smoke condensate reduces calcium activated chloride channel transport in primary sinonasal epithelial cultures. Laryngoscope. 2010; 120:1465-1469. [PubMed: 20564721]

15. Wanner A, Salathe M, O'Riordan TG. Mucociliary clearance in the airways. Am J Respir Crit Care Med. 1996; 154:1868-1902. [PubMed: 8970383]

16. Kreindler JL, Jackson AD, Kemp PA, Bridges RJ, Danahay H. Inhibition of chloride secretion in human bronchial epithelial cells by cigarette smoke extract. Am J Physiol Lung Cell Mol Physiol. 2005; 288:L894-L902. [PubMed: 15626749]

17. Antunes MB, Woodworth BA, Bhargave G, et al. Murine nasal septa for respiratory epithelial airliquid interface cultures. Biotechniques. 2007; 43:195-196. 198, 200 passim. [PubMed: 17824387]

18. Bhargave G, Woodworth BA, Xiong G, Wolfe SG, Antunes MB, Cohen NA. Transient receptor potential vanilloid type 4 channel expression in chronic rhinosinusitis. Am J Rhinol. 2008; 22:712. [PubMed: 18284852]

19. Woodworth BA, Tamashiro E, Bhargave G, Cohen NA, Palmer JN. An in vitro model of Pseudomonas aeruginosa biofilms on viable airway epithelial cell monolayers. Am J Rhinol. 2008; 22:234-238.

20. Virgin F, Zhang S, Schuster D, et al. The bioflavonoid compound, sinupret, stimulates transepithelial chloride transport in vitro and in vivo. Laryngoscope. 2010; 120:1051-1056. [PubMed: 20422703]

21. Azbell C, Zhang S, Skinner D, Fortenberry J, Sorscher EJ, Woodworth BA. Hesperidin stimulates cystic fibrosis transmembrane conductance regulator-mediated chloride secretion and ciliary beat 
frequency in sinonasal epithelium. Otolaryngol Head Neck Surg. 2010; 143:397-404. [PubMed: 20723778]

22. Zhang S, Fortenberry JA, Cohen NA, Sorscher EJ, Woodworth BA. Comparison of vectorial ion transport in primary murine airway and human sinonasal air-liquid interface cultures, models for studies of cystic fibrosis, and other airway diseases. Am J Rhinol Allergy. 2009; 23:149-152. [PubMed: 19401039]

23. Pyle, L.; Ehrhardt, A.; Nowotarski, K.; Rowe, SM.; Sorscher, EJ. North American Cystic Fibrosis Foundation Annual Meeting. Denver, CO: 2006. The role of R-domain phosphorylation in cftr activation by potentiators.

24. Eiserich JP, van der Vliet A, Handelman GJ, Halliwell B, Cross CE. Dietary antioxidants and cigarette smoke-induced biomolecular damage: a complex interaction. Am J Clin Nutr. 1995; 62:1490S-1500S. [PubMed: 7495250]

25. O'Toole TE, Zheng YT, Hellmann J, Conklin DJ, Barski O, Bhatnagar A. Acrolein activates matrix metalloproteinases by increasing reactive oxygen species in macrophages. Toxicol Appl Pharmacol. 2009; 236:194-201. [PubMed: 19371603]

26. Cantin AM, Hanrahan JW, Bilodeau Get al. Cystic fibrosis transmembrane conductance regulator function is suppressed in cigarette smokers. Am J Respir Crit Care Med. 2006; 173:1139-1144. [PubMed: 16497995] 


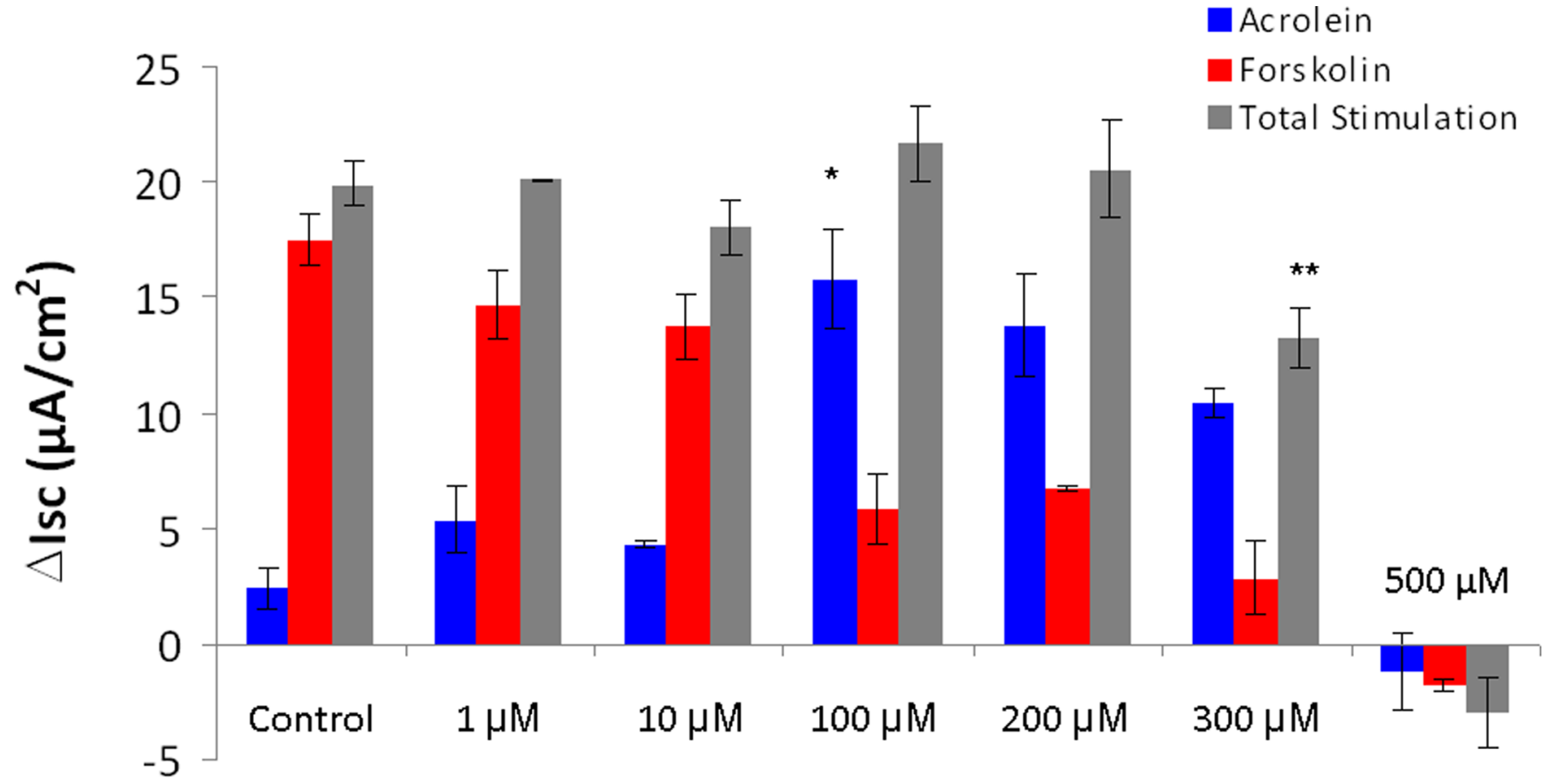

Acrolein Concentration

$$
\begin{aligned}
{ }^{*} \mathrm{P} & <0.0001 \\
{ }^{* *} \mathrm{P} & <0.01
\end{aligned}
$$

Figure 1. Impact of acrolein on transepithelial $\mathrm{Cl}^{-}$secretion is concentration dependent Maximal $\Delta \mathrm{I}_{\mathrm{SC}}$ was noted at $100 \mu \mathrm{M}(\mathrm{p}<0.0001)$, but decreased with increasing concentrations of compound. Total stimulation supplemented with forskolin was significantly inhibited at $300 \mu \mathrm{M}(\mathrm{p}<0.01)$ and completely abolished with exposure to 500 $\mu \mathrm{M}$ of acrolein. 


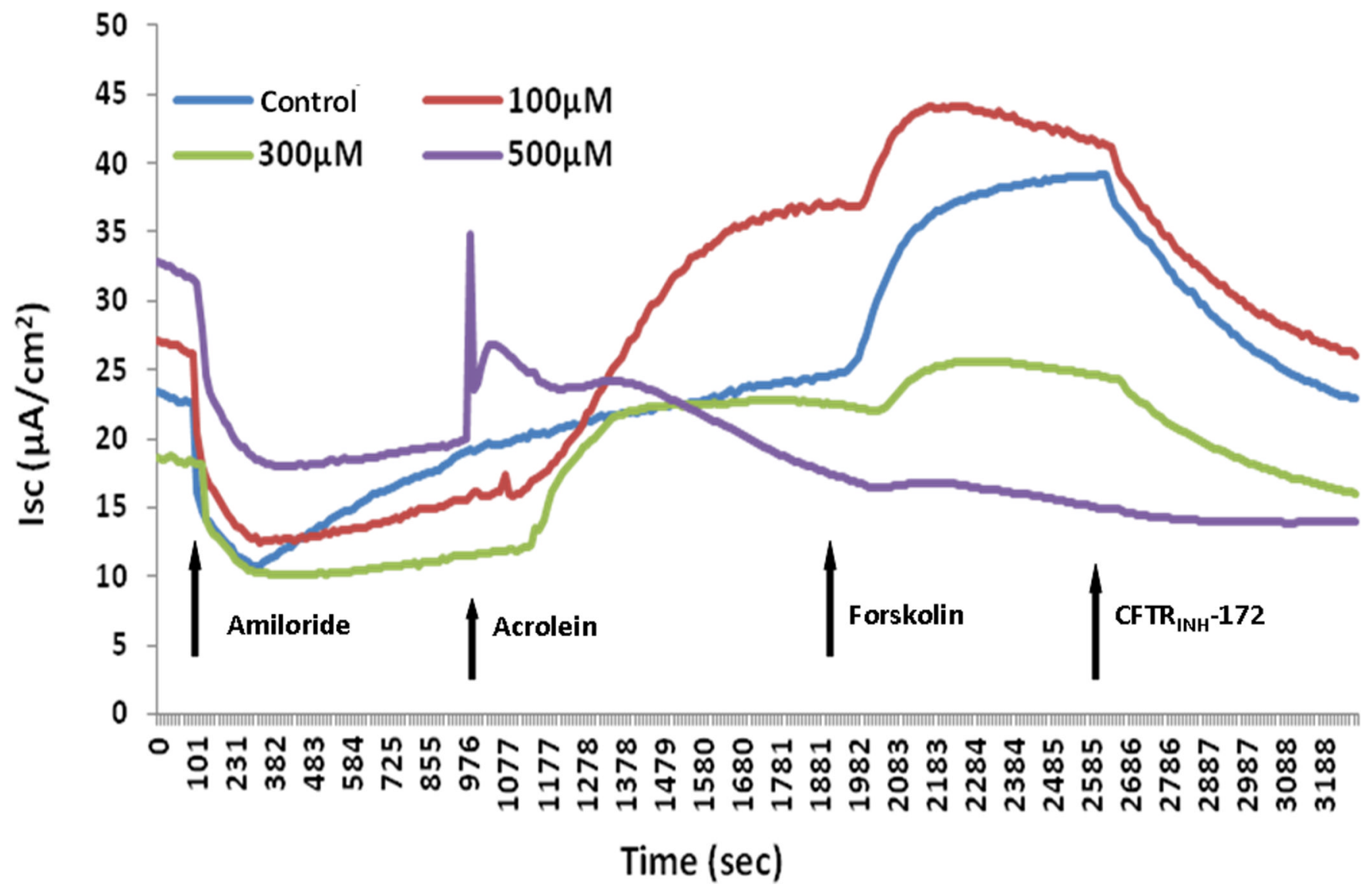

Figure 2. Representative Ussing chamber tracings of wild type MNSE cultures treated with acrolein

Wild type MNSE cells grown on transwell permeable supports were mounted in Ussing chambers under short-circuit conditions and sequentially exposed to amiloride, acrolein, forskolin, and $\mathrm{CFTR}_{\mathrm{INH}^{-}}$172. A positive deflection indicates the net movement of an anion from the serosal to the mucosal direction. Note the paradoxical decrease in total $\Delta \mathrm{I}_{\mathrm{SC}}$ at higher concentrations. 


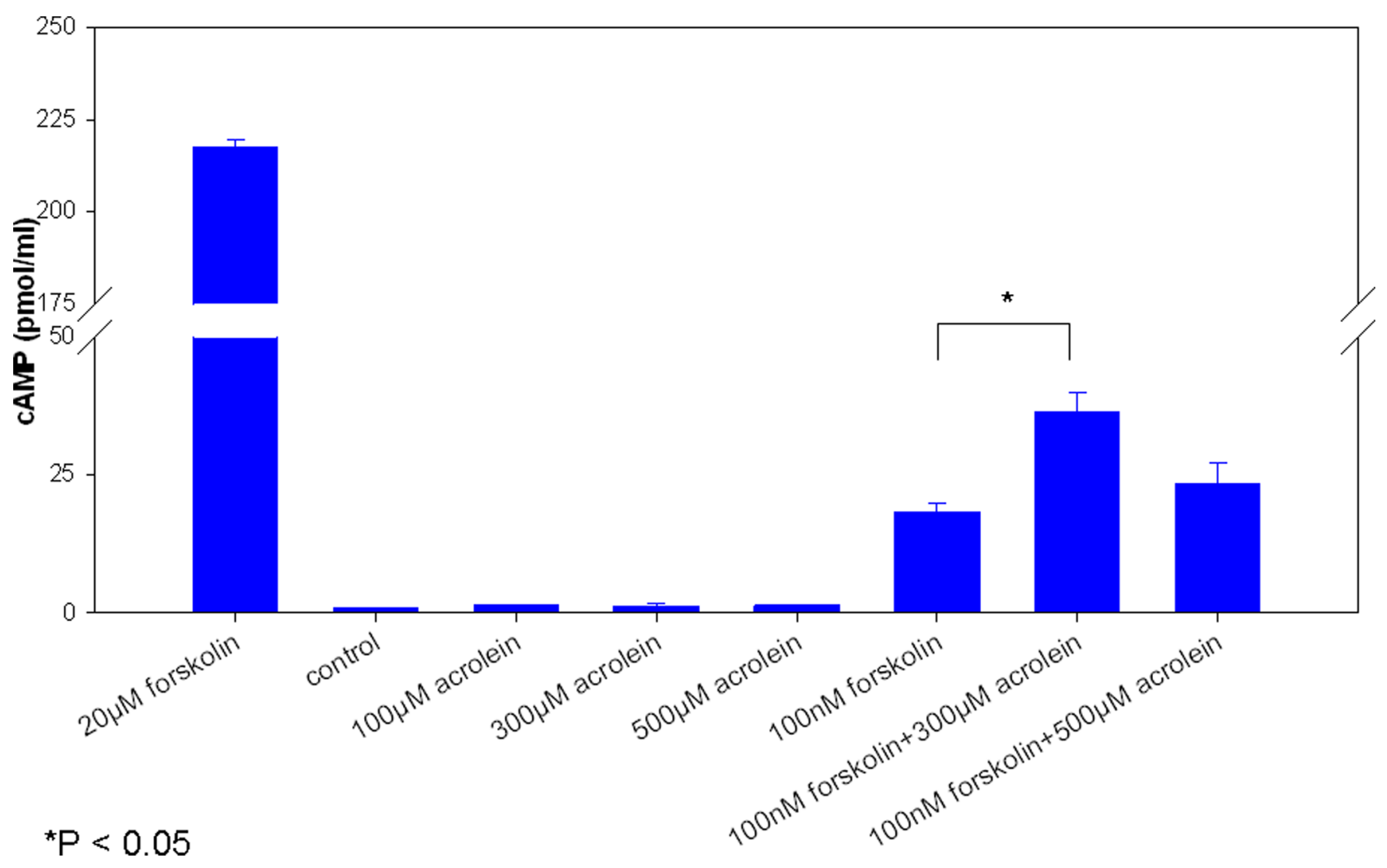

Figure 3. Ussing chamber analysis of $100 \mu \mathrm{M}$ acrolein exposure

While maximal $\Delta \mathrm{I}_{\mathrm{SC}}$ was noted at this concentration $(\mathrm{p}<0.0001)$, measurements of total stimulated (acrolein + forskolin) and CFTR inhibited $\left(\mathrm{CFTR}_{\mathrm{INH}^{-}}{ }^{172}\right) \Delta \mathrm{I}_{\mathrm{SC}}$ revealed no significant differences, suggesting near maximal stimulation of CFTR by acrolein alone. 


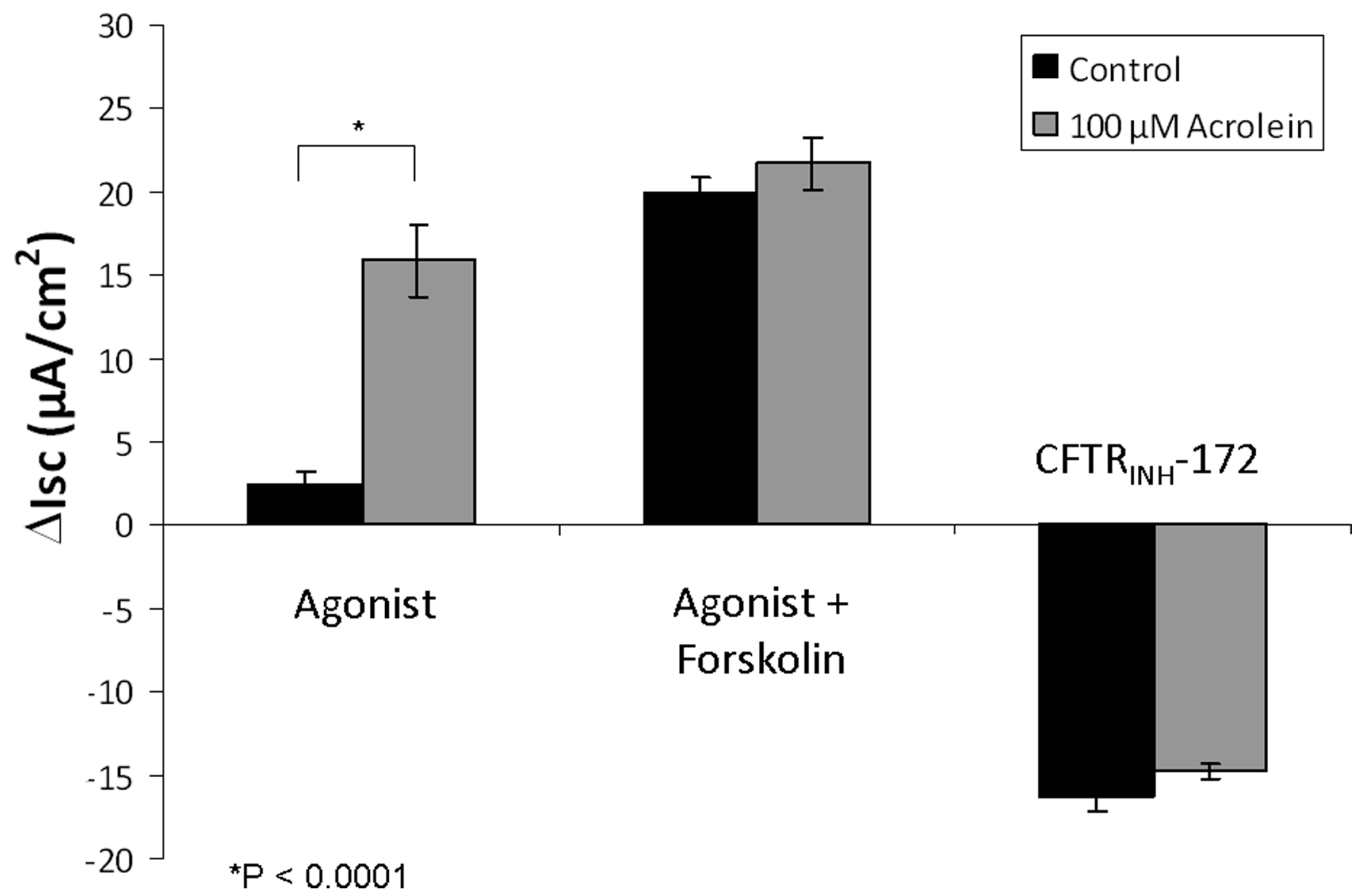

Figure 4. Representative Ussing chamber tracings of transgenic CFTR $^{-/-}$MNSE cultures treated with $100 \mu \mathrm{M}$ acrolein

No significant increase in $\Delta \mathrm{I}_{\mathrm{SC}}$ was measured signifying effects on apical $\mathrm{Cl}^{-}$transport are CFTR dependent. 


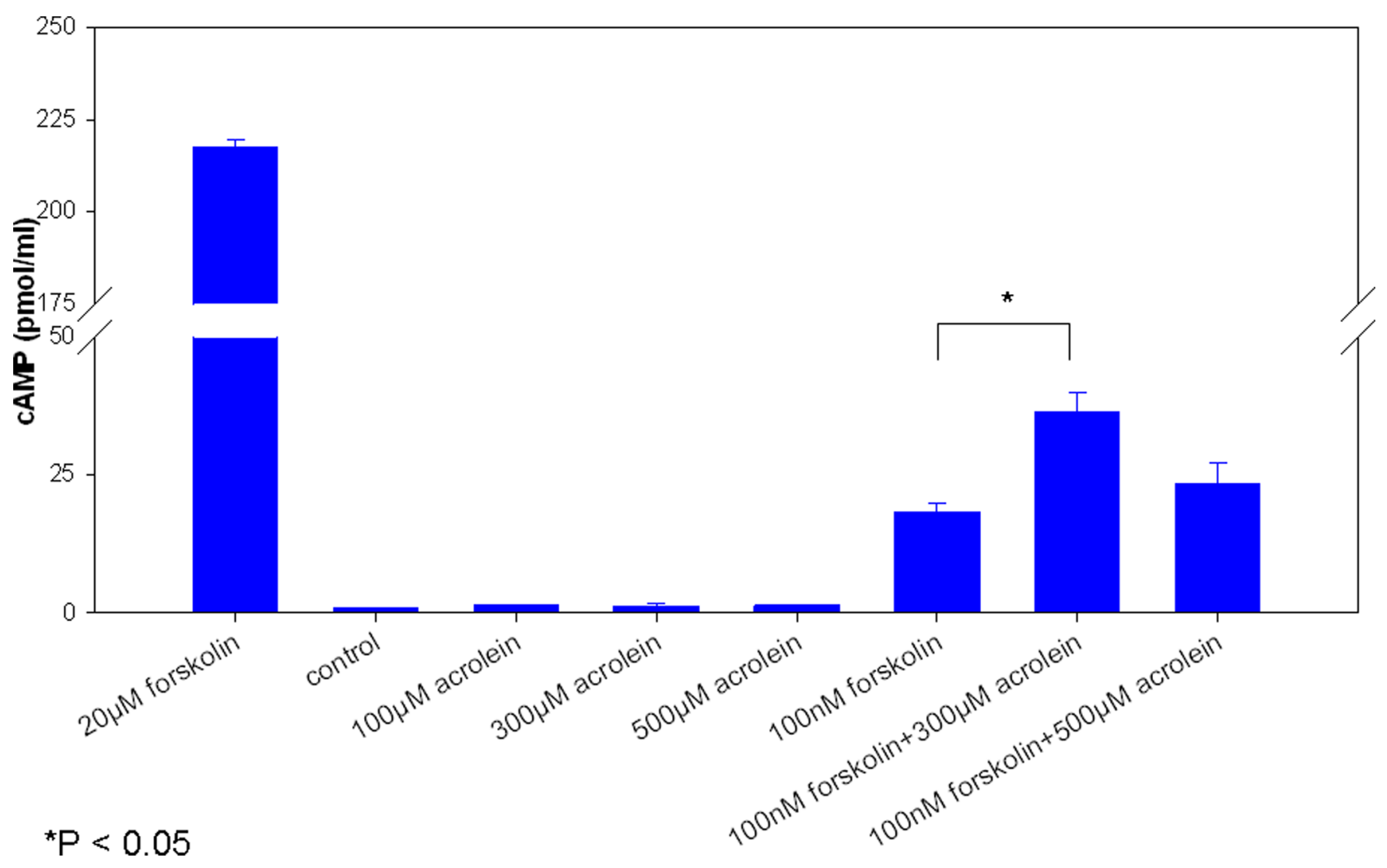

Figure 5. Acrolein's impact on CFTR is independent of cAMP stimulation

No significant stimulation was demonstrated with graded concentrations of acrolein alone. Pre-incubation of cultures with higher doses of acrolein $(300$ and $500 \mu \mathrm{M})$ did not inhibit forskolin activation of adenylyl cyclase. A significant increase was seen over forskolin alone with the $300 \mu \mathrm{M}$ concentration, but this is biologically insignificant. 